\title{
Incubation period as part of the case definition of severe respiratory illness caused by a novel coronavirus
}

H Nishiura (nishiura@hku.hk)1,2, K Mizumoto ${ }^{1,3}, \mathrm{~K} \mathrm{Ejima}^{1,4}$, Y Zhong $^{1}$, B J Cowling ${ }^{1}$, R Omori ${ }^{1}$

1. School of Public Health, The University of Hong Kong, Hong Kong SAR, China

2. PRESTO (Precursory Research for Embryonic Science and Technology), Japan Science and Technology Agency, Saitama, Japan

3. Institute of Tropical Medicine and the Global Center of Excellence Program, Nagasaki University, Nagasaki, Japan

4. Department of Mathematical Informatics, Graduate School of Information Science and Technology, The University of Tokyo, Tokyo, Japan

Citation style for this article:

Nishiura H, Mizumoto K, Ejima K, Zhong Y, Cowling BJ, Omori R. Incubation period as part of the case definition of severe respiratory illness caused by a novel coronavirus. Euro Surveill. 2012;17(42):pii=20296. Available online: http://www.eurosurveillance.org/ViewArticle.aspx?Articleld=20296

Non-specific symptoms of acute respiratory viral infections make it difficult for many countries without ongoing transmission of a novel coronavirus to rule out other possibilities including influenza before isolating imported febrile individuals with a possible exposure history. The incubation period helps differential diagnosis, and up to two days is suggestive of influenza. It is worth including the incubation period in the case definition of novel coronavirus infection.

\section{Introduction}

Two cases of severe respiratory infection have been confirmed as caused by a novel coronavirus [1]. The case definition has been issued by the World Health Organization (WHO), and is mainly based on acute respiratory illness, pneumonia (or suspicion of pulmonary parenchymal disease) and travel history [2]. To describe the clinical characteristics of the novel coronavirus infection, the incubation period has played a key role in suspecting Saudi Arabia and Qatar as geographic locations of exposure for the two cases mentioned above $[1,3]$. The presumed length of the incubation period was compared with known incubation periods of human coronavirus infections including that of severe acute respiratory syndrome (SARS) $[3,4]$. The present study intends to point out that the incubation period can be useful for all countries without ongoing transmission to distinguish the novel coronavirus infection from other viral respiratory infections, most notably influenza.

\section{Methods}

\section{Motivating case study}

A preschool child from Saudi Arabia was admitted to a Hong Kong hospital equipped with an isolation ward in early October 2012, suspected of novel coronavirus infection. It had fever, cough and vomiting, but did not have pneumonia. One close contact had had a fever two days earlier, but had recovered before the day of admission [5]. Assuming that the contact was the source of infection, the serial interval was two days, which is typically longer than the incubation period
$[6,7]$, and thus, the incubation period is likely to have been two days or shorter. On the day following admission, the child tested negative for the novel coronavirus, but positive for influenza $A\left(\mathrm{H}_{1} \mathrm{~N}_{1}\right)$ pdmog [5].

A similar event, but involving two cases of severe pneumonia, occurred in Denmark: A cluster of febrile patients, some of whom had a travel history to Qatar and Saudi Arabia, was suspected of infection with the novel coronavirus. However, later laboratory testing revealed that the respiratory illnesses were caused by infection with an influenza B virus [8].

We believe that the distinction between coronavirus and influenza virus infections in these settings could have been facilitated by considering the length of the incubation period.

\section{Bayesian model}

Let $f_{\mathrm{i}}\left(t \mid \theta_{\mathrm{i}}\right)$ be the probability density function of the incubation period $t$ of virus $i$ governed by parameter $\theta_{\mathrm{i}}$. The incubation period distributions for a variety of acute upper respiratory viral infections have been fitted to log-normal distributions elsewhere $[4,9]$ and are assumed known hereafter. The median incubation periods of SARS, non-SARS human coronavirus infection, and influenza $A$ and influenza $B$ virus infections have been estimated at 4.0, 3.2, 1.4 and 0.6 days, respectively [4]. It should be noted that the median incubation periods of influenza $A$ and $B$ have been estimated as shorter than those of coronaviruses. The incubation period $f_{\mathrm{i}}$ is assumed to be independent across different viruses $i$. Due to shortage of information, we ignore the time-dependence and geographic heterogeneity in the risk of infection for all viruses. The posterior probability of novel coronavirus infection (which is labelled as $i=1$ ) given an incubation period $t, \operatorname{Pr}$ (novel coronavirus $\mid t)$ is then obtained by using a Bayesian approach:

$\operatorname{Pr}($ novel coronavirus $\mid t)=\frac{q_{1} f_{1}\left(t \mid \theta_{1}\right)}{\sum_{i} q_{i} f_{i}\left(t \mid \theta_{i}\right)}$ 
where $q_{i}$ denotes the prior probability of virus $i$ (e.g. $q_{1}=\operatorname{Pr}$ (novel coronavirus); the probability that the novel coronavirus is responsible for acute respiratory viral infection with unknown aetiology among all such infections), which can be equated to the relative frequency of virus $i$ infection during a viral aetiological study (e.g. using the relative incidence by aetiological agent) $[10,11]$. Since the observed data are recorded on a daily basis, the incubation period in (1) is discretised as,

$f_{i, t} \Rightarrow \int_{0}^{t} f_{i}\left(s \mid \theta_{1}\right) d s-\int_{0}^{t-1} f_{i}\left(z \mid \theta_{1}\right) d z$

for $t>0$.

Since the prior probability $q_{i}$ is unknown for imported cases with acute respiratory illness, two conservative approaches, which would not lead to an underestimation of the probability of novel coronavirus infection, should be taken. Such approaches include (i) allocating an equal probability as the prior probability for all possible viruses (e.g. for a differential diagnosis of two viral diseases, we allocate 0.5 for each) or (ii) using results from published viral aetiological studies among people with an acute respiratory disease (e.g. using virus detection results among influenza-like illness (ILI) patients). As an example for the latter approach, the observed numbers of coronavirus infections and influenza $A$ and $B$ virus infections among $177 \mathrm{ILI}$ cases in children with known viral aetiology have been 12, 40 and 5 cases, respectively, in Madagascar [12]. Here we focus on this particular dataset among children only, because the case in Hong Kong, whom we want to use to exemplify our theoretical idea, was of preschool age. Moreover, we used the data from Madagascar, because this study appeared informative as it closely investigated the frequency of different types of human coronaviruses among ILI cases in children [12]. It should be noted that $n=12$ in Madagascar does not represent the frequency of novel coronavirus infections, but the frequency of infections caused by other human coronaviruses, while the estimation of the posterior probability of novel coronavirus infection using equation (1) requires the prior probability of the novel coronavirus. Here we use this figure for the novel coronavirus, for the purposes of presenting of our theory.

\section{Results}

The Figure (panel A) shows the conditional probability of coronavirus infection given the incubation period (based on equation (1)), in a setting where one has to differentiate coronavirus infection from influenza virus infection, assuming an equal probability of 0.5 for either virus. Assuming that the observed incubation period of the child in Hong Kong was two days, the probability of non-SARS human coronavirus infection is smaller than $0.1 \%$. When using the incubation period of SARS as a reference to represent the incubation period of novel coronavirus, the probability of the coronavirus infection with a two-day incubation period is $15.7 \%$. In other words, the probability of influenza A given a twoday incubation period is as high as $99.9 \%$ and $84.3 \%$, respectively, when comparing between influenza $A$ and either non-SARS or SARS coronaviruses. Various control measures, including case isolation, contact tracing and laboratory testing can make use of this probability (e.g. contact tracing may assume that new generations of cases would arise on average every three days, consistent with influenza transmission). A calculation for influenza B virus yielded qualitatively similar results (Figure, panel A).

It should be noted that the actual relative frequency of novel coronavirus is much smaller than that discussed here, due to the absence of substantial human-tohuman transmission events [3], while influenza A virus has already circulated in the human population. Thus, the posterior probability of novel coronavirus in reality would be much smaller than that illustrated in the Figure.

When we use the empirically observed frequency of human coronaviruses based on the viral aetiological study data among ILI cases in children (Figure, panel $B)$, the probabilities of coronavirus and influenza $A$ and $B$ virus are estimated at $<0.1 \%, 65.7 \%$ and $1.4 \%$, respectively. It is remarkable that an ILI with the incubation period of two days is most likely to be caused by influenza A virus. However, novel coronavirus may be suspected if the incubation period is in the order of three to five days.

\section{Discussion}

As demonstrated in this report, the probability of infection with novel coronavirus can be inferred from the incubation period of each single case with suspected infection, which we believe is useful for deciding on a public health alert level and the extent of movement restriction and contact tracing among imported cases of acute respiratory viral infection, especially with mild and non-specific symptoms. We have shown that an incubation period of two days or shorter is strongly suggestive of influenza, while an incubation period from three to five days could potentially be consistent with the incubation period of human coronaviruses. Of course, the implementation of isolation measures, contact tracing and other interventions would also depend on other factors including the perceived importance and cost of the interventions, but we have shown at least that the incubation period would yield supplementary information for differential diagnosis and decision making. We believe that it is worth considering incorporating the incubation period into the case definition as soon as sufficient data on the incubation period have been collected.

In practice, the proposed approach suits case investigations (or outbreak investigations) in which precise information of contacts is collected, because estimates of the incubation period are often available. However, 
A

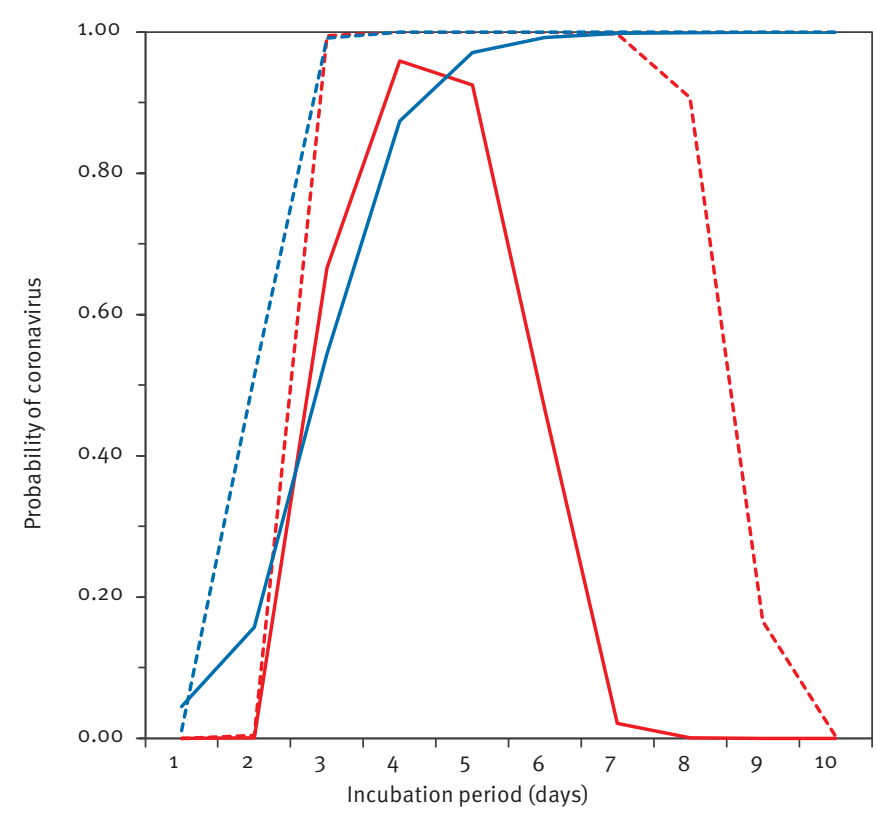

- HCoV vs influenza A
---- HCoV vs influenza B
B
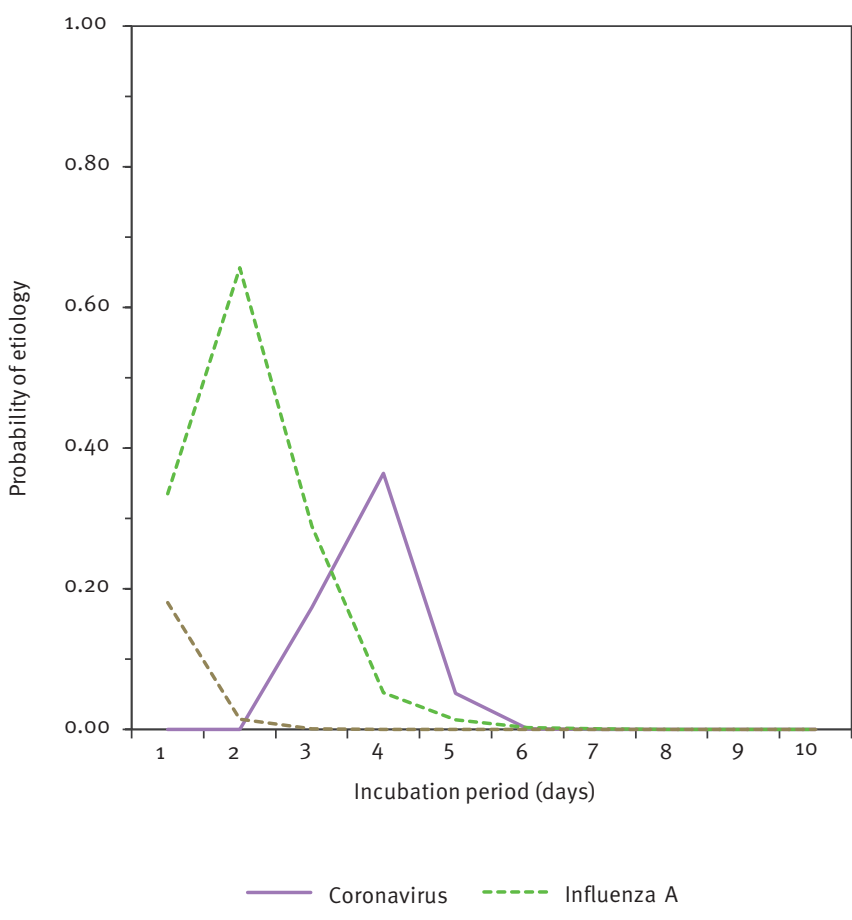

A. The probability of coronavirus infection given the incubation period, when comparing between coronavirus infection and influenza virus infection as possible diagnoses. We use $50 \%$ probability for each of the two viruses (i.e. coronavirus versus influenza virus) for a conservative argument to avoid an underestimation of the risk of novel coronavirus. Since known coronaviruses are classified into severe acute respiratory syndrome (SARS)-associated virus and non-SARS viruses, and because influenza viruses are crudely classified as type A and $B$ viruses, there are four possible combinations for comparison. HCoV stands for human coronavirus infection other than severe acute respiratory syndrome (SARS).

B. The probability of coronavirus infection given the incubation period, using empirically observed viral aetiology data as a prior information among influenza-like illness cases in Madagascar [12] with a total of $n=177$ samples for those aged younger than five years. The observed number of isolates, i.e. Influenza A $(\mathrm{n}=40)$, Influenza B $(\mathrm{n}=5), \mathrm{HCoV}(\mathrm{n}=12)$ and others $(\mathrm{n}=120)$, were used to calculate $q_{\mathrm{i}}$ in equation $(1)$. $\mathrm{n}=12$ for ordinary $\mathrm{HCoV}$ is here used as if it gave the frequency of a novel coronavirus, for the purpose pf presenting our theory. The incubation periods of viruses other than influenza viruses and human coronaviruses were assumed to be uniformly distributed from day 1 to day 10, for a conservative argument to avoid an underestimation of the probability of novel coronavirus.

three common technical issues should be discussed. Firstly, as an infection event cannot be directly observed, multiple contacts can limit straightforward information on an incubation period. For instance, we cannot technically rule out the possibility that the child case in Hong Kong was exposed to someone other than the close contact before travelling to Hong Kong. Secondly, the incubation period tends to be crude, especially for the first few cases, e.g. when the length of travel with an exposure is long for imported cases. Thirdly, one cannot guarantee that the incubation period of a novel pathogen is always similar to that of closely related pathogens. For instance, the incubation period of Escherichia coli $0104: \mathrm{H}_{4}$ infection has been

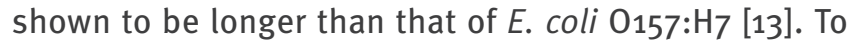
address the second and third point, it is essential to collect multiple datasets of the incubation period with a brief exposure.

In addition to its value in differential diagnosis, considering the incubation period has important public health implications. Firstly, to help differential diagnosis during the course of an epidemic of any novel infectious disease, the distribution should be estimated as early as possible. For this reason, the detailed travel history of imported cases should be explored, as it can inform the distribution of incubation periods $[9,14]$. Moreover, outbreak reports, including case reports, should explicitly and routinely document the detailed history of exposure (e.g. the length and timing of exposure along with the illness onset date) of all cases. Secondly, the overall risk estimate (e.g. the relative 
incidence) would be essential to validate the proposed Bayesian model (1), although in reality, the prior probability varies considerably with time and place. To understand the ongoing risk of infection with a novel virus explicitly, a population-wide serological survey, which allows to infer at least the cumulative incidence, would be a useful method to offer insights into the aetiology. Finally, while estimating the relative probability of alternative aetiologies can help with diagnosis, decisions on possible control measures (such as isolation of cases) could also be affected by other concerns including reduction in the risk of larger outbreaks.

\section{Acknowledgments}

The work of HN was supported by the JST PRESTO program and St Luke's Life Science Institute Research Grant for Clinical Epidemiology Research 2012. KE received scholarship support from the Japan Society for Promotion of Science (JSPS). This work also received financial support from the Harvard Center for Communicable Disease Dynamics from the National Institute of General Medical Sciences (grant no. U54 GMo88558).
References

1. Danielsson N, On Behalf of The Ecdc Internal Response Team C, Catchpole M. Novel coronavirus associated with severe respiratory disease: Case definition and public health measures. Euro Surveill. 2012;17(39):pii=20282. Available from: http://www.eurosurveillance.org/ViewArticle. aspx?Articleld $=20282$

2. World Health Organization. Global alert and response (GAR): revised interim case definition-novel coronavirus. Geneva, Switzerland: World Health Organization; 2012. [Accessed 11 Oct 2012]. Available from: http://www.who.int/csr/disease/ coronavirus_infections/case_definition/en/index.html

3. Pebody RG, Chand MA, Thomas HL, Green HK, Boddington $\mathrm{NL}$, Carvalho C, et al. The United Kingdom public health response to an imported laboratory confirmed case of a novel coronavirus in September 2012. Euro Surveill. 2012;17(40): pii=20292. Available from: http://www. eurosurveillance.org/ViewArticle.aspx?Articleld =20292

4. Lessler J, Reich NG, Brookmeyer R, Perl TM, Nelson KE, Cummings DA. Incubation periods of acute respiratory viral infections: a systematic review. Lancet Infect Dis 2009;9(5):291-300.

5. Saudi boy in Hong Kong has flu, not Sars-like virus. Hong Kong: South China Morning Post; 8 Oct 2012. Available from: http://www.scmp.com/news/hong-kong/article/1056321/ saudi-boy-tests-negative-sars-virus-hong-kong-healthofficials-say

6. Nishiura H, Eichner M. Infectiousness of smallpox relative to disease age: estimates based on transmission network and incubation period. Epidemiol Infect. 2007;135(7):1145-50.

7. Klinkenberg D, Nishiura H. The correlation between infectivity and incubation period of measles, estimated from households with two cases. J Theor Biol. 2011;284(1):52-60.

8. Patient i højrisiko-isolation er ikke smittet med coronavirus. [Patient in high-risk isolation is not infected with coronavirus]. Hvidovre: Hvidovre Hospital. [Accessed 16 Oct 2012]. Danish. Available from: http://www.hvidovrehospital.dk/menu/ Presse+og+nyheder/Nyheder+fra+Hvidovre+Hospital/2012/Pa tient+i+hjrisikoisolation+er+ikke+smittet+med+Coronavirus. htm?wbc_purpose=Basictar

9. Nishiura $\mathrm{H}$, Inaba $\mathrm{H}$. Estimation of the incubation period of influenza A ( $\mathrm{H}_{1} \mathrm{~N}_{1}$-2009) among imported cases: addressing censoring using outbreak data at the origin of importation. J Theor Biol. 2011;272(1):123-30.

10. Nishiura H. Incubation period as a clinical predictor of botulism: analysis of previous izushi-borne outbreaks in Hokkaido, Japan, from 1951 to 1965 . Epidemiol Infect. 2007;135(1):126-30.

11. Lessler J, Brookmeyer R, Perl TM. An evaluation of classification rules based on date of symptom onset to identify health-care-associated infections. Am J Epidemiol. 2007;166(10):1220-9.

12. Razanajatovo NH, Richard V, Hoffmann J, Reynes JM, Razafitrimo GM, Randremanana RV, et al. Viral etiology of influenza-like illnesses in Antananarivo, Madagascar, July 2008 to June 2009. PLoS One. 2011;6(3):e17579.

13. Buchholz U, Bernard H, Werber D, Böhmer MM, Remschmidt C, Wilking $\mathrm{H}$, et al. German outbreak of Escherichia coli $0_{104}: \mathrm{H}_{4}$ associated with sprouts. N Engl J Med. 2011;365(19):1763-70.

14. Nishiura H, Lee HW, Cho SH, Lee WG, In TS, Moon SU, et al. Estimates of short- and long-term incubation periods of Plasmodium vivax malaria in the Republic of Korea. Trans R Soc Trop Med Hyg. 2007;101(4):338-43. 